\title{
Segregationsprozesse beim Übergang vom Elementarbereich in die Grundschule. Wie verändern Wanderungsbewegungen zwischen den Schulbezirken Kompositionen in Bezug auf Migrationshintergrund?
}

\author{
Johanna Gold • Jan Christoph Störtländer • Philipp Dierker • \\ Annette Textor
}

Eingegangen: 30. Oktober 2020 / Angenommen: 19. April 2021 / Online publiziert: 21. Januar 2022 (C) Der/die Autor(en) 2022

Zusammenfassung Der staatliche Bildungsauftrag sieht vor, dass junge Menschen durch die Schule die Verwirklichungschance zu gesellschaftlicher Teilhabe unabhängig von Herkunft oder Lebenslage erhalten. Dieses Versprechen wird gleichzeitig vom deutschen Bildungssystem permanent enttäuscht. Dafür liegt nicht erst seit PISA starke empirische Evidenz vor.

Insbesondere bei Übergängen zwischen Bildungseinrichtungen kommt es zu Segregationsprozessen, bei denen Zugangs- und Verwirklichungschancen zu Bildungsoptionen ungleich verteilt werden. So verstärkt das Bildungssystem soziale Disparitäten oder bringt sie gar hervor. Gut erforscht ist der Übergang vom Primar- in den Sekundarbereich. Er gilt als Gelenkstelle für Segregationsprozesse aufgrund individueller Lebenslagen. Auch auf den Übergang vom Elementar- in den Primarbereich trifft das zu. Auf Segregation ausgelegte Schulstrukturen vermindern die Partizipationschancen für Kinder aus sozioökonomisch benachteiligten Elternhäusern und solchen mit Migrationshintergrund.

Der vorliegende Beitrag zeichnet nach, wie Segregationsprozesse im Bildungssystem am Übergang zwischen dem Elementarbereich und der Primarstufe zu Homogenisierungsprozessen der Schülerschaft hinsichtlich des Merkmals Ethnizität führen. Auf Grundlage empirischer Daten eines Einschulungsjahrgangs $(n=3180)$ wird beispielhaft ein Schulgeflecht auf die Veränderung von Kompositionen hin untersucht. Mögliche Auswirkungen auf Partizipation und Inklusion werden aus der Perspektive des Capability Approach diskutiert.

Schlüsselwörter Kompositionen · Wanderungsbewegungen zwischen Schulbezirken $\cdot$ Segregationsprozesse $\cdot$ Schulgeflecht $\cdot$ Schulwahl

Johanna Gold $(\varangle) \cdot$ Jan Christoph Störtländer · Philipp Dierker · Annette Textor Universität Bielefeld, Bielefeld, Deutschland

E-Mail: johanna.gold@uni-bielefeld.de 


\title{
Segregation processes in the transition from pre-primary to elementary school. How do inter-district movements change compositions in terms of migration background?
}

\begin{abstract}
School as a central social institution shapes the capabilities of young people to a large extent. Educational policies and mandates aim at social participation of students regardless of their origin and living situation. At the same time, however, there is strong empirical evidence - and not just since the PISA studies-that this very promise is constantly being disappointed by the German education system.

Thus, on the basis of precisely this empirical evidence, there is a consensus in social science discourse that the education system reinforces or even creates social disparities. A main problem is social segregation by transitions between educational institutions. A main focus of previous research on segregation based on social circumstances is the transition between primary school and lower secondary school. Recent research turns to the prior transition from elementary education to primary education. Again, these analyses show that segregating school structures particularly affects the participation opportunities for students from socioeconomically disadvantaged homes or homes with a migrant background.

This article analyses segregation processes at the transition between the elementary and primary level based on empirical data of a school enrollment cohort $(n=3180)$. The results show homogenization processes with regard to ethnicity within the population of so called 'school-nets'-i.e. single schools and their surrounding neighborhoods particularly affected by composition effects. The results are discussed from a capabilities approach perspective with special consideration of disadvantages that impede participation and inclusion.
\end{abstract}

Keywords Compositions $\cdot$ Movement between school districts $\cdot$ Segregation processes $\cdot$ School web $\cdot$ School choice

\section{Einführung}

Die Zusammensetzung der Schülerschaft in deutschen Schulen und Schulklassen variiert stark hinsichtlich bestimmter individueller und sozialer Merkmale der Schüler*innen. Häufungen bestimmter Merkmale, insbesondere von sozial benachteiligenden Lebenslagen, führen zu besonderen pädagogischen Herausforderungen und nehmen Einfluss auf Schulleistungen und daraus abgeleitet auf die Bildungschancen der Schüler*innen. Phänomen hat unter dem Begriff Kompositionseffekt Eingang in den Diskurs der Bildungsforschung gefunden. Kompositionseffekte werden im erziehungswissenschaftlichen Diskurs als der Einfluss der Zusammensetzung der Schüler*innen eines Klassenverbandes im Hinblick auf sozio-kulturelle und ethnisch-kulturelle Merkmale, soziale Risikofaktoren, das Fähigkeits- und Leistungsniveau und lernbiographische Belastungsfaktoren der Kinder auf ihre Schulleistung und ihre Bildungsbeteiligung beschrieben. Von Kompositionseffekten wird gesprochen, wenn diese zusammengefassten individuellen Merkmale aller Schüler*innen einer Klasse (oder Schule) zusätzlich zu den individuellen Merkmalen eines Kindes 
einen Einfluss auf Schulleistungen und Bildungsbeteiligung entfalten. Insbesondere bei Schüler*innen aus belasteten Lebenslagen können sich so zusätzliche, schwerwiegende Nachteile ergeben (Dumont et al. 2013).

Dieser Beitrag fokussiert die Komposition einer Schule im Hinblick auf ethnisch-kulturelle Merkmale und zeichnet nach, wie durch Ab- und Zuwanderung die Komposition im Vergleich zur Ausgangspartition des Schulbezirks verändert wird. Ethnisch-kulturelle Merkmale finden insbesondere unter dem Begriff Migrationshintergrund - in der Regel operationalisiert über die primäre Sprache im Haushalt oder die Geburtsländer der Eltern und/oder Kinder - Eingang in zahlreiche Studien zur Bildungsbenachteiligung. Diese kommen übereinstimmend zu dem Ergebnis, dass ein negativer Effekt dieses Merkmals auf Schulleistung und Bildungsbeteiligung vorliegt (Autorengruppe Bildungsberichterstattung 2016, S. $173 \mathrm{ff}$.), der nach Kontrolle des sozioökonomischen Status der Schüler*innen deutlich reduziert ist (Stanat et al. 2010; Rjosk et al. 2017). Während entsprechende strukturbezogene Analysen für den Übergang von der Primarstufe in die Sekundarstufe I vorliegen, wurde der Übergang vom Elementar- in den Primarbereich bisher eher unter einer subjektorientierten Perspektive betrachtet (Hogrebe 2016). Der vorliegende Beitrag zeichnet vor diesem Hintergrund nach, wie Segregationsprozesse im Bildungssystem am Übergang zwischen Elementarbereich und der Primarstufe zu Homogenisierungsprozessen der Schülerschaft entlang der Dimension der ethnisch-kulturellen Merkmale führen. Dabei wird auf Grundlage empirischer Daten eines Einschulungsjahrgangs $(n=3180)$ ein beispielhaftes Schulgeflecht auf die Veränderung von Kompositionen hin untersucht. Anschließend werden aus der Perspektive des Capability Approaches und aus habitustheoretischer Perspektive die daraus entstehenden Nachteile, die Partizipation und Inklusion verhindern können, aufgezeigt.

\section{Theoretische Einordnung}

\subsection{Bildungswege und Befähigungswege}

Die Befunde zu Bildungsdisparitäten und insbesondere jene zu mehr oder weniger in elterlicher Hand liegenden Wahlmöglichkeiten bei Eintritt in das Pflichtschulwesen werfen zum einen aus gerechtigkeitstheoretischer Perspektive die Fragen auf, inwiefern die Liberalisierung der Grundschulwahl grundsätzlich ein an Bildung und Teilhabe orientiertes Moment der politischen Steuerung des Bildungswesens ist; dies ist eine Frage, die in diesem Unterabschnitt kurz theoretisch konturiert wird. Zum anderen werfen sie die damit zusammenhängende empirische Frage auf, welche Nutzung von Wahlmöglichkeiten wir exemplarisch feststellen können und welche Annahmen sich daraus hinsichtlich unseres Verständnisses von Bildungsgerechtigkeit generieren lassen; dies wird im weiteren Verlauf unseres Beitrags untersucht.

Unsere gerechtigkeitstheoretische Perspektive und damit auch unser Verständnis von Bildungsgerechtigkeit geht aus dem Capabilities Approach (dt. „Befähigungs- 
ansatz") ${ }^{1}$ hervor. Dessen prominenteste Vertreter*innen Amartya Sen (2011) und Martha Nussbaum (2007) entwickeln in neu-aristotelischer Tradition eine Heuristik, mit der sie menschliches Gedeihen (human flourishing) betrachten. Die Grundfrage ist dabei, ob und welche realen Freiheiten und Verwirklichungschancen unter konkreten sozialen Bedingungen bestehen, sodass Menschen in der Lage sind oder in die Lage versetzt werden, dasjenige Leben zu führen, das sie berechtigterweise wertschätzen, mithin ein Leben führen zu können, das der menschlichen Würde gerecht wird.

Der Zugang zu Bildung ist in diesem Verständnis einer der zentralen Faktoren in der Gestaltung der gesellschaftlichen Rahmenbedingungen, die ein gelingendes Leben befördern können. Hierbei ,schwebt [dem Aristoteliker] die Kombination eines mehr oder weniger einheitlichen öffentlichen Erziehungssystems mit einer flexibleren privaten Erziehung in der und durch die Familie vor" (Nussbaum 2016, S. 79). Nussbaum verweist hier exemplarisch auf die Sozial- und Bildungssysteme (nord-)europäischer Staaten. Capabilities-basierte empirische Studien legen dabei immer wieder offen, dass der (freie) Zugang zu öffentlichen Bildungseinrichtungen und deren faktische Nutzung durch die Bürger*innen zu einer erheblichen Verbesserung der individuellen Befähigungssituation führt und das Ausbleiben oder Verschließen dieses Zugangs sich in einer ,zersetzenden Benachteiligung“ mit zum Teil drastischer negativer Auswirkung auf die gesamte Befähigungssituation von Menschen äußert (Wolff und de-Shalit 2007). Uns scheint eine Capabilities-orientierte Perspektive auf Bildungswege und deren Ex- bzw. Inklusivität unter einem zweifachen Verständnis von Mobilität gewinnbringend zu sein.

Erstens gehen wir davon aus, dass Bildungswege soziale Mobilität eröffnen oder verschließen und dass dies den beteiligten Akteur*innen weitestgehend bewusst ist. Diese soziale Mobilität verstehen wir nicht in Sinne eines irgendwie gearteten „,Aufstiegs“", sondern betrachten sie unter der Frage, ,wie Bildung Individuen (besonders aus sozial deprivierten Bevölkerungsgruppen) in ihrem Emanzipationsprozess aus einer kulturell, politisch oder ökologisch bedingten Unmündigkeit unterstützen kann“ (Gold 2018, S. 287). Dabei haben wir Grund zu der Annahme, dass jedenfalls Schüler*innen am Ende ihrer Schullaufbahn dieses Verständnis von Mobilität nicht nur teilen, sondern auch eigenständig artikulieren (Störtländer 2019). Dabei sind sowohl die entwickelten Vorstellungen von Bildung als auch die soziale Mobilität selber milieuspezifisch konturiert (Aytekin und Boger 2016) und ihnen wird von institutioneller Seite mehr oder weniger förderlich begegnet (Boger und Störtländer 2019).

Diese Überlegungen verweisen darauf, dass bei der Betrachtung von sozialer Mobilität durch Bildung, Bildungserwerb und Bildungszugang in dem Verhältnis von öffentlichem Erziehungssystem und privater Erziehung in und durch die Familie ein Spannungsfeld von Passungsverhältnissen zwischen diesen beiden Instanzen liegt. Genauer beleuchtet werden kann dieses Spannungsfeld mit Hilfe der Habitus-Theorie. Habitus wird verstanden als ,Erzeugungsprinzip objektiv klassifizierbarer Formen von Praxis und Klassifikationssystem dieser Formen“ (Bourdieu 1982, S. 277).

\footnotetext{
${ }^{1}$ Zitate aus englischsprachigen Publikationen wurden von Jan Christoph Störtländer ins Deutsche übersetzt.
} 
Das Konstrukt dient somit als Erklärungsansatz für den Einfluss von Lebensbedingungen auf individuell verschiedene Haltungen, Gewohnheiten und Kompetenzen und die daraus resultierenden Auswirkungen auf Handlungsweisen.

Zweitens hat Mobilität eine sehr konkrete physische Komponente. Nussbaum weist als eine zentrale menschliche Befähigung die Möglichkeit aus ,sich frei von Ort zu Ort bewegen zu können“ (Nussbaum 2011, S. 32). Nun sind unsere deutschen Städte von keinen (sichtbaren) Barrieren durchzogen, aber wir kennen bei den Grundschulbezirken das Prinzip „kurze Beine - kurze Wege“; d.h. ein Grund, die Schüler*innen in ihren Bezirken zu beschulen, ist die möglichst fußläufige Erreichbarkeit einer Grundschule. Mit der Liberalisierung der Schulwahl wird dieses Prinzip grundsätzlich außer Kraft gesetzt und es ist zu vermuten, dass eine Verschickung des Kindes in einen anderen Schulbezirk nur von denjenigen Eltern in Erwägung gezogen wird, die sich das leisten bzw. die das organisieren können.

So stehen wir vor der ambivalenten Situation, auf der einen Seite mit der Liberalisierung und grundsätzlichen Eröffnung der Grundschulwahl eine Forderung des Capabilities Approach eingelöst zu haben: nämlich die Forderung, die eigenen (elterlichen) Vorstellungen von Bildung für das Kind ohne den Zwang des Schulbezirks realisieren zu können. Auf der anderen Seite vermuten wir eine Zunahme von Segregation, die aus eben dieser Perspektive Anlass zu Kritik und Sorge gibt.

\subsection{Inklusion und Teilhabe}

Der Anspruch auf gleichberechtigten Zugang zu Bildung ist eng verknüpft mit der Forderung eines inklusiven - übersetzt als nicht-diskriminierenden - Bildungssystems. Ein ,auf alle Lernenden, besonders aber auf vulnerable Gruppen bezogenes Adressatenverständnis“ (Lindmeier und Lütje-Klose 2015, S. 8) von Inklusion schließt dabei die unterschiedlichen Dimensionen gesellschaftlicher Deprivilegierung $^{2}$ sowie die Berücksichtigung von Phänomenen der Intersektionalität ein. In diesem Artikel konzentrieren wir uns auf Kinder, denen ein Migrationshintergrund zugeschrieben wird, wohl wissend, dass diese Zuschreibung häufig mit Zuschreibungen auf der Fähigkeitsdimension (,,ability“33) sowie mit Benachteiligungen im sozioökonomischen Bereich (,class“) einhergeht. Gleichzeitig werden die Förderschwerpunkte Lernen, Sprache und emotionale und soziale Entwicklung Kindern mit Migrationshintergrund trotz der Unterschiede im Kompetenzniveau nicht signifikant häufiger zugeschrieben (Kölm et al. 2017). Anders ist dies hinsicht-

\footnotetext{
2 Da wir von der Annahme ausgehen, dass Vulnerabilität eine anthropologische Konstante ist - auch wenn Risiken sowie die Ressourcen zum Umgang mit diesen gesellschaftlich ungleich verteilt sind - und da sich unsere Analysen auf der Systemebene bewegen und sich daher letztendlich mit der Frage nach gesellschaftlichen Prozessen von (De)Privilegierung und Marginalisierung beschäftigen, verwenden wir anstelle des eher individualisierenden Begriffs der ,,(besonders) vulnerablen Gruppen“ den der ,deprivilegierten“ oder „,marginalisierten“ Gruppen, da dieser deutlicher die bedingenden gesellschaftlichen Strukturen benennt.

3 Das in der internationalen Literatur verwendete Pendant - „Ability“ bzw., wenn die Diskriminierung im Vordergrund steht, ,Ableism“ - wird häufig mit „Behinderung“ bzw. „Behindertenfeindlichkeit“ übersetzt; diese Übersetzung ist jedoch unzureichend. Die Kategorie „Ableism“ bezeichnet Diskriminierungsprozesse gegenüber Personen, denen weniger (marktkonforme) Leistungsfähigkeit zugeschrieben wird. Dies kann, aber muss nicht infolge einer Behinderung sein (Boger und Textor 2016, S. $84 \mathrm{ff}$.).
} 
lich des Besuchs einer Förderschule: „Kinder aus Familien mit einem im Ausland geborenen Elternteil besuchen häufiger eine Förderschule als Kinder mit zwei im Ausland geborenen Elternteilen und als Kinder ohne Zuwanderungshintergrund“ (Kölm et al. 2017, S. 298). Insgesamt ist es aufgrund dieser engen statistischen Zusammenhänge zwischen Migrationshintergrund, sozioökonomischem Status und Leistungsstand sinnvoll, von einem weiten Inklusionsbegriff auszugehen, der alle Dimensionen gesellschaftlicher Deprivilegierung einbezieht.

Der Anspruch auf Inklusion als diskriminierungsfreien und gleichberechtigten Zugang zu Bildung lässt sich einerseits aus dem Gleichheitsgrundsatz demokratischer Gesellschaften ableiten (Maaz 2017), andererseits wird mit einem inklusionsorientierten Schulsystem die Hoffnung verbunden, dass mit einem solchen demokratisches Lernen befördert werden könne. Inklusion und Teilhabe schließt so „eine bewusste, demokratisch geprägte Positionierung“ (Textor 2018, S. 35), gegenüber Diskriminierungen und Partizipationseinschränkungen sensibel zu sein, diese $\mathrm{zu}$ verringern und sich mit betroffenen Personen zu solidarisieren, ein.

\subsection{Empirische Erkenntnisse zur Segregation im Übergang vom Elementarbereich in die Primarstufe}

20 Jahre nach der Veröffentlichung der PISA 2000-Ergebnisse herrscht im erziehungs- und sozialwissenschaftlichen Diskurs, weithin Konsens darüber, dass das Bildungssystem soziale Disparitäten hervorbringt oder verstärkt, wobei deren Genese ,sich als Folge sozialschichtabhängiger Entscheidungsprozesse an den Gelenkstellen von Bildungskarrieren verstehen“ lässt (Maaz et al. 2010, S. 23). Der Übergang von der Primarstufe in die Sekundarstufe I wird in diesem Zusammenhang als „Schlüsselübergang“ gekennzeichnet (Maaz 2017). So greift auch die Grundschule bestehende soziale Disparitäten auf und fungiert eher als „Chancenverteilerin“ (van Ackeren und Klemm 2019, S. 405 ff.) denn als Angleicherin oder Ausgleicherin von Chancen. Allerdings ist davon auszugehen, dass bereits der Eintritt in das Schulsystem - der Übergang vom Elementar- in den Primarbereich - zu sozialen Segregationen führt.

Eine Segregation der Schülerschaft, die über die residentielle Segregation hinausgeht und diese verschärft, ist bereits zu beobachten, wenn verbindliche Schulbezirke bestehen (Fincke und Lange 2012; Makles et al. 2019) - beispielsweise indem eine Bekenntnisschule, eine internationale Schule oder eine Schule in freier Trägerschaft angewählt wird. Vermutet werden könnte, dass Segregationsprozesse umso mehr zu finden sind, wenn verbindliche Schuleinzugsgebiete aufgehoben werden, die Wahl der Grundschulen freigestellt wird und so ein „Quasi-Markt“ (Clausen 2006, S. 72) entsteht. Dies scheint aber empirisch nicht der Fall zu sein: Eine Studie, die die Segregation vor und nach der Freigabe der Grundschulwahl in NRW in Bezug auf ethnische Gruppen vergleicht, findet zwar eine Steigerung der ethnischen Segregation vor, diese konnte jedoch bereits vor Auflösung der Schulbezirke beobachtet werden (Makles und Schneider 2013).

Gleichzeitig zeigt sich in der Studie von Hogrebe (2016), die die soziale und ethnische Segregation in Einrichtungen des Elementarbereiches und des Primarbereiches mithilfe von Daten aus der Schuleingangsuntersuchung vergleicht, dass „Kinder 
mit Migrationshintergrund und Kinder ohne elterliche Förderung ein vergleichbares Ausmaß an Segregation erfahren, das im Elementarbereich stärker ausgeprägt ist als an Grundschulen. Besonders betroffen sind aber Kinder von Eltern, die über unzureichende Sprachkenntnisse verfügen“"(Hogrebe 2016, S. 104). Inwiefern Wahlmöglichkeiten mit Segregationsprozessen einhergehen, bleibt vor dem Hintergrund der bisherigen Forschungslage somit unklar; einerseits finden Segregationsprozesse auch in Regionen mit offiziell sehr limitierten Wahlmöglichkeiten statt, andererseits ist die Segregation im Elementarbereich, dessen Einrichtungen von den Eltern aktiv angewählt werden müssen, höher als im Primarbereich. Insgesamt kann also festgestellt werden, dass der Befund, dass Eltern (sowie deren Kinder) im Übergang von der Primarstufe in die Sekundarstufe I ihren Bildungsaspirationen folgend weitreichende Bildungsentscheidungen unter kontingenten Bedingungen treffen (Köller et al. 2019), ebenso für den Übergang vom Elementar- in den Primarbereich gilt.

Krüger et al. (2020) gehen auf Basis ihrer multiperspektivischen Studien zu ,Szenarien der Grundschulwahl" vor diesem Hintergrund davon aus, dass sich Eltern und Erziehungsberechtigte von einzuschulenden bzw. schulpflichtig werdenden Kindern in einem komplexen Geflecht von (teilweise verdeckten) Entscheidungsoptionen und Entscheidungsnotwendigkeiten befinden, das sie in einer Situation der Unentscheidbarkeit, Unabgeschlossenheit und Unberechenbarkeit zurücklässt, aus der heraus Bildungsentscheidungen bei der Wahl der Grundschule getroffen werden.

Aus diesen theoretischen Vorannahmen und empirischen Befunden heraus stellen wir uns die Frage, ob und in welche Richtung die unter den Bedingungen freier Schulwahl möglichen Wanderungsbewegungen zwischen den Schulbezirken die Kompositionen der Einzelschulen verändern. Diese Fragestellung bearbeiten wir anhand von Daten des Bildungsbüros der Stadt Bielefeld, die im Prozess von der Schulanmeldung bis zur Einschulung entstehen.

\section{Design der Studie}

\subsection{Beschreibung des Feldes}

Das Forschungsprojekt Schulwahl und Bildung (SchuB) untersucht in Kooperation mit den am Einschulungsprozess beteiligten Ämtern den Prozess der Einschulung vor dem Hintergrund der Aufhebung der Verbindlichkeit der Grundschulbezirke. Es fokussiert die Zusammenhänge von divergenten Lebenslagen der Familien sowie

Tab. 1 Übersicht über die Bindung der Grundschulen an Schulbezirke und die Möglichkeiten der inklusiven Beschulung

\begin{tabular}{|c|c|c|c|}
\hline Bezirksbindung & Anzahl & Inklusive Beschulung & Anzahl \\
\hline \multirow{2}{*}{$\begin{array}{l}\text { An einen Schulbezirk gebundene } \\
\text { städtische Schulen }\end{array}$} & \multirow[t]{2}{*}{43} & Schulen mit GL & 19 \\
\hline & & Schulen ohne GL & 24 \\
\hline \multirow{2}{*}{$\begin{array}{l}\text { An keinen Schulbezirk gebundene } \\
\text { Schulen in kirchlicher, privater oder } \\
\text { sonstiger Trägerschaft }\end{array}$} & \multirow[t]{2}{*}{7} & $\begin{array}{l}\text { Ausgewiesene inklusive Beschu- } \\
\text { lung }\end{array}$ & 3 \\
\hline & & $\begin{array}{l}\text { Keine ausgewiesene inklusive } \\
\text { Beschulung }\end{array}$ & 4 \\
\hline
\end{tabular}


deren Dispositionen auf der einen und Schulwahl sowie Schulzuweisung auf der anderen Seite. mit dem Ziel, Anhaltspunkte für ein umfassendes Modell zur Schulwahl im Übergang vom Elementar- in den Primarbereich zu erhalten, das sowohl Motive der Schulwahl und Prozesse der Präferenzbildung als auch Prozesse der Realisierung der Schulwahl enthält.

Die Schullandschaft Bielefelds besteht im Primarbereich aus 50 Grundschulen und elf Förderschulen mit einer Primarstufe. Tab. 1 stellt die Anzahl der bezirksgebundenen und nicht bezirksgebundenen Schule und die Möglichkeit der inklusiven Beschulung dar. Sieben der 50 Grundschulen befinden sich nicht in städtischer sondern in kirchlicher, privater oder sonstiger Trägerschaft. Das Inklusionskonzept der Stadt sieht vor, Kinder mit einem festgestellten sonderpädagogischen Förderbedarf an ausgewiesenen Schulen des Gemeinsamen Lernens (GL), zu bündeln. Zurzeit sind 19 der 43 Schulen in städtischer Trägerschaft im Primarbereich GL-Schulen. Von den Schulen, die nicht in städtischer Trägerschaft sind, weisen zusätzlich drei Schulen explizit eine inklusive Beschulung in ihrem Schulprogramm aus.

In der hier beschriebenen Stadt ist jeder städtischen Grundschule ein Schulbezirk zugeordnet; Schulen in nicht-städtischer Trägerschaft sowie Förderschulen haben keinen Schulbezirk. Die Bezirke der städtischen Grundschulen sind nicht bindend Wie viele und welche Kinder in einem Schulbezirk leben ist somit nicht deckungsgleich damit, wie viele und welche Kinder die jeweilige Schule besuchen.

\subsection{Datenbasis}

Die hier vorliegende Analyse basiert auf einem Datensatz, der aus administrativen Daten der städtischen Schulstatistik generiert wurde und setzt sich aus 3180 Schüler*innen zusammen, die im Schuljahr 2018/19 eingeschult wurden. Es liegen personenscharf sowohl die Zugehörigkeit zum Einzugsbezirk als auch die Angabe der aufnehmenden Schule wie auch Angaben zum Migrationshintergrund und zum Geschlecht der Schüler*innen vor. Die Angaben zum Migrationshintergrund werden in den Schulbüros generiert und basieren auf den von den Eltern erteilten Informationen zur Erstsprache im Elternhaus.

Für acht der 3180 Kinder liegt keine gesicherte Information über die Zugehörigkeit zum Schulbezirk vor, fünf Kinder sind während des Anmeldeverfahrens für die Grundschule noch nicht in Bielefeld wohnhaft gewesen. Die Wohnorte der 3167 Kinder, für die Informationen zur Bezirkszugehörigkeit vorliegen, verteilen sich zahlenmäßig ungleichmäßig auf die 43 städtischen Einzugsbezirke. Die Besetzung der Bezirke liegt zwischen 34 und 151.

Für den tatsächlichen Besuch an der Grundschule zeigt sich ein etwas anderes Bild: 69 Kinder aus den städtischen Wohnbezirken wurden außerhalb des Stadtgebiets eingeschult, 37 Kinder sind aus der Stadt weggezogen. Die Stärke der Einschulungsjahrgänge liegt zwischen 23 und 96 Kindern.

Tab. 2 gibt einen Überblick über die 3143 auf Schulen im Stadtbezirk eingeschulten Kinder. An den 43 städtischen Schulen sind 89,4\% (2843 Kinder) des gesamten Jahrganges, eingeschult worden. Rund $47 \%$ davon besuchen eine städtische Schule mit Gemeinsamem Lernen. 7,2\% (226 Kinder) wurden an Schulen angenommen, die nicht in städtischer Trägerschaft liegen. Von diesen Kindern besuchen rund $58 \%$ ei- 
Tab. 2 Übersicht über die Einschulungsquoten aufgeteilt nach Art der Schule und Möglichkeit der inklusiven Beschulung

\begin{tabular}{|c|c|c|c|}
\hline Art der Schule & $\begin{array}{l}\text { Anzahl der aufgenommenen } \\
\text { Kinder } \\
\text { (in Klammern: Anteile be- } \\
\text { zogen auf den Gesamtjahr- } \\
\text { gang) }\end{array}$ & $\begin{array}{l}\text { Inklusive } \\
\text { Beschulung }\end{array}$ & $\begin{array}{l}\text { Anzahl der aufgenommenen } \\
\text { Kinder } \\
\text { (in Klammern: Anteile be- } \\
\text { zogen auf den Gesamtjahr- } \\
\text { gang) }\end{array}$ \\
\hline \multirow{2}{*}{$\begin{array}{l}\text { Städtische } \\
\text { Grundschulen } \\
\text { (mit Bindung an } \\
\text { Bezirk) }\end{array}$} & \multirow[t]{2}{*}{$2843(89,4 \%)$} & $\begin{array}{l}\text { Schulen mit } \\
\text { GL }\end{array}$ & $1344(42,3 \%)$ \\
\hline & & $\begin{array}{l}\text { Schulen ohne } \\
\text { GL }\end{array}$ & $1499(47,1 \%)$ \\
\hline $\begin{array}{l}\text { Schulen in } \\
\text { kirchlicher, } \\
\text { privater oder }\end{array}$ & \multirow[t]{2}{*}{$226(7,2 \%)$} & $\begin{array}{l}\text { Ausgewiesene } \\
\text { inklusive } \\
\text { Beschulung }\end{array}$ & $132(4,2 \%)$ \\
\hline $\begin{array}{l}\text { Sonstiger } \\
\text { Trägerschaft } \\
\text { (ohne Bindung an } \\
\text { Bezirk) }\end{array}$ & & $\begin{array}{l}\text { Keine aus- } \\
\text { gewiesene } \\
\text { inklusive Be- } \\
\text { schulung }\end{array}$ & $94(3,0 \%)$ \\
\hline $\begin{array}{l}\text { Förderschulen } \\
\text { (ohne Bindung an } \\
\text { Bezirk) }\end{array}$ & $5(0,2 \%)$ & - & - \\
\hline $\begin{array}{l}\text { Einschulung auf } \\
\text { Schulen außerhalb } \\
\text { des Stadtgebietes }\end{array}$ & $69(2,2 \%)$ & - & - \\
\hline Wegzug & $37(1,2 \%)$ & - & - \\
\hline
\end{tabular}

ne Schule mit ausgewiesener inklusiver Beschulung. An einer Förderschule wurden fünf Kinder des Jahrganges eingeschult (s. Tab. 2).

$67,8 \%$ der Kinder des Einschulungsjahrganswurden in der ihrem Schulbezirk zugehörigen Schule eingeschult, 32,2\% besuchen eine Schule außerhalb ihres Schulbezirks. Demnach hat rund ein Drittel der Familien der schulpflichtigen Kinder dieses Jahrgangs eine Entscheidung für einen anderen als den eigenen Schulbezirk getroffen und konnte diese Entscheidung auch umsetzen. Es zeigt sich allgemein kein signifikanter Unterschied in den Wechselbewegungen in Abhängigkeit davon, ob die Schule des eigenen Bezirks eine GL-Schule oder nicht ist. Aus Bezirken mit GL-Schulen wandern 32,6\% der Kinder auf Schulen außerhalb dieser Bezirke ab, in Bezirken mit Schulen ohne GL liegt dieser Anteil bei 31,7\%. Es liegen im Rahmen des hier verwendeten Datensatzes keine Informationen darüber vor, wie viele Kinder in einem anderen Schulbezirk angemeldet wurden, aber dort nicht aufgenommen wurden.

Im folgenden Kapitel stellen wir auf Grundlage der vorgestellten Datenbasis deskriptive Auswertungen der Veränderungen der Kompositionen vor und nach der Schulwahl mit einem Fokus auf das Merkmal Migrationshintergrund vor. 


\section{Ergebnisse}

\subsection{Deskriptive Darstellung der besonders von Wanderungsbewegungen und Homogenisierungseffekten betroffenen Schulen und Einzugsbezirken.}

In den folgenden Datenanalysen dient der von der aufnehmenden Schule gemeldete Migrationshintergrund der Kinder als das Merkmal, anhand dessen die Segregationsbzw. Homogenisierungseffekte aufgezeigt werden. Dieses steht in engem Zusammenhang mit den Heterogenitätsdimensionen ,class“ und ,,ability“ (s. Abschn. 2.2), zu denen jedoch bisher keine Daten vorliegen. Die Darstellung der Daten erfolgt deskriptiv. Tab. 3 gibt eine Übersicht über den Anteil der Kinder in den Bezirken und in den bezirksgebundenen Schulen, für die ein Migrationshintergrund gemeldet wird denen durch die aufnehmende Schule somit eine nicht ausschließlich deutsche Ethnizität zugeschrieben wird. Sowohl in den Bezirken wie auch in den Schulen variieren die Anteile stark. Die Spannweite bei den Schulbezirken bewegt sich zwischen 0,0 und 73,8\% (Mittelwert $=24,0 \%$, Standardabweichung=19,5). Bei den bezirksgebundenen Schulen bewegen sich diese Anteile zwischen 0,0 und 86,7\% (Mittelwert $=26,6^{4} \%$, Standardabweichung $=27,7$ ), während die Anteile bei den Schulen in nicht-städtischer Trägerschaft zwischen 0,0 und 36,7\% liegen. Die Differenzen zwischen den Anteilen der Kinder mit einem zugeschriebenen Migrationshintergrund, die in den jeweiligen Bezirken gemeldet sind, und den Anteilen der Kinder, die nach den Wanderungsbewegungen auf der Schule des Bezirks eingeschult werden, variieren ebenfalls sehr stark. Sie liegen zwischen einer Reduktion des Anteils der Kinder mit Migrationshintergrund um 19,4 Prozentpunkte und einem Zuwachs dieses Anteils um 26,6 Prozentpunkte. Tab. 3 ist auch zu entnehmen, auf wie viele verschiedene Schulen sich aus dem Bezirk abwandernde Kinder verteilen.

Jede Schule in städtischer Trägerschaft ist genau einem Einzugsbezirk zugeordnet. Die Anzahl der einem Schulbezirk zugeordneten Kinder und die Anzahl der Kinder, die die Schule des Bezirks besuchen, sind jedoch durch die Möglichkeit der freien Schulwahl nicht deckungsgleich. Die vorhandenen Daten erfassen, welcher Anteil von Kindern den zugewiesenen Schulbezirk verlässt und welcher Anteil der Kinder auf jeder Schule aus einem oder mehreren anderen Schulbezirk(en) rekrutiert wird. Die Schulbezirke sind nicht gleichmäßig von Wanderungsbewegungen betroffen. Tab. 4 zeigt eine Auflistung der sechs Bezirke, die besonders von Abwanderung zu anderen Schulen betroffen sind. Die höchste beobachtete Abwanderungsquote aus einem Schulbezirk liegt bei 65,8\%. Auffällig ist hierbei Schule S-17: Ungefähr die Hälfte der Kinder wandert aus dem Schulbezirk ab, etwas mehr als die Hälfte der Schulanfänger wandert aus anderen Bezirken zu, ein solcher gravierender Austausch der Schüler*innen ist in keinem anderen Bezirk zu beobachten.

\footnotetext{
${ }^{4}$ Der Unterschied im Mittelwert zwischen den Bezirken und Schulen erklärt sich dadurch, dass die Kinder, die eine nicht bezirksgebundene Schule besuchen im Mittelwert der Bezirke auftauchen aber nicht im Mittelwert der bezirksgebundenen Schulen.
} 
Tab. 3 Übersicht über die Anzahl der in den Bezirken gemeldeten Kinder, die Größe der Einschulungsjahrgänge und die Anteile der von der Schule gemeldeten Schüler*innen mit Migrationshintergrund in den Schulbezirken und den Schulen und die Anzahl der alternativ angewählten Schulen bei Abwanderung

\begin{tabular}{|c|c|c|c|c|c|c|}
\hline $\begin{array}{l}\text { Schul- } \\
\text { bezirk/ } \\
\text { Schule }\end{array}$ & $\begin{array}{l}\text { Anzahl } \\
\text { gemel- } \\
\text { deter } \\
\text { Kinder }\end{array}$ & $\begin{array}{l}\text { Anteil der } \\
\text { Kinder mit } \\
\text { Migrations- } \\
\text { hintergrund } \\
\text { im Bezirk } \\
(\%)\end{array}$ & $\begin{array}{l}\text { Anzahl } \\
\text { einge- } \\
\text { schulter } \\
\text { Kinder }\end{array}$ & $\begin{array}{l}\text { Anteil der Kin- } \\
\text { der mit Migrati- } \\
\text { onshintergrund } \\
\text { in der Schule } \\
(\%)\end{array}$ & $\begin{array}{l}\text { Differenz } \\
\text { der Quo- } \\
\text { ten (in } \\
\text { Prozent- } \\
\text { punkten) }\end{array}$ & $\begin{array}{l}\text { Anzahl der } \\
\text { alternativ } \\
\text { angewähl- } \\
\text { ten Schulen }\end{array}$ \\
\hline$\overline{S-23}$ & 62 & 19,4 & 59 & 0,0 & $-19,4$ & 5 \\
\hline$S-16-G L$ & 123 & 12,2 & 82 & 0,0 & $-12,2$ & 14 \\
\hline$S-32$ & 112 & 11,6 & 74 & 0,0 & $-11,6$ & 13 \\
\hline$S-13$ & 68 & 8,8 & 96 & 0,0 & $-8,8$ & 5 \\
\hline$S-37$ & 79 & 10,1 & 78 & 2,6 & $-7,5$ & 9 \\
\hline$S-45$ & 45 & 8,9 & 35 & 2,9 & $-6,0$ & 6 \\
\hline$S-18$ & 35 & 8,6 & 74 & 2,7 & $-5,9$ & 5 \\
\hline$S-8$ & 34 & 5,9 & 23 & 0,0 & $-5,9$ & 6 \\
\hline$S-9$ & 57 & 5,3 & 53 & 0,0 & $-5,3$ & 6 \\
\hline$S-38-G L$ & 109 & 20,2 & 83 & 15,7 & $-4,5$ & 11 \\
\hline$S-12$ & 51 & 5,9 & 55 & 1,8 & $-4,1$ & 6 \\
\hline S-39 & 87 & 19,5 & 90 & 15,6 & $-3,9$ & 8 \\
\hline$S-15-G L$ & 49 & 6,1 & 45 & 2,2 & $-3,9$ & 6 \\
\hline$S-43-G L$ & 65 & 21,5 & 73 & 17,8 & $-3,7$ & 4 \\
\hline$S-46-G L$ & 79 & 8,9 & 38 & 5,3 & $-3,6$ & 8 \\
\hline$S-14$ & 42 & 31,0 & 68 & 27,9 & $-3,1$ & 4 \\
\hline$S-42$ & 57 & 7,0 & 60 & 5,0 & $-2,0$ & 5 \\
\hline$S-36-G L$ & 106 & 28,3 & 78 & 26,9 & $-1,4$ & 12 \\
\hline$S-22-G L$ & 151 & 1,3 & 89 & 0,0 & $-1,3$ & 9 \\
\hline$S-44$ & 82 & 0,0 & 83 & 0,0 & 0,0 & 7 \\
\hline$S-50$ & 57 & 8,8 & 43 & 9,3 & 0,5 & 8 \\
\hline$S-40-G L$ & 89 & 18,0 & 75 & 18,7 & 0,7 & 14 \\
\hline$S-4-G L$ & 41 & 7,3 & 79 & 8,9 & 1,6 & 4 \\
\hline$S-34-G L$ & 119 & 1,7 & 85 & 3,5 & 1,8 & 8 \\
\hline$S-3$ & 96 & 36,5 & 87 & 39,1 & 2,6 & 7 \\
\hline$S-31$ & 58 & 1,7 & 36 & 5,6 & 3,9 & 5 \\
\hline$S-2-G L$ & 51 & 45,1 & 72 & 50,0 & 4,9 & 4 \\
\hline$S-28-G L$ & 86 & 22,1 & 96 & 28,1 & 6,0 & 8 \\
\hline$S-41$ & 64 & 17,2 & 37 & 24,3 & 7,1 & 12 \\
\hline$S-7-G L$ & 80 & 47,5 & 80 & 55,0 & 7,5 & 8 \\
\hline$S-1$ & 55 & 50,9 & 51 & 58,8 & 7,9 & 4 \\
\hline$S-49$ & 84 & 29,8 & 66 & 37,9 & 8,1 & 8 \\
\hline$S-47-G L$ & 80 & 50,0 & 78 & 59,0 & 9,0 & 11 \\
\hline$S-11-G L$ & 52 & 44,2 & 69 & 53,6 & 9,4 & 6 \\
\hline$S-29-G L$ & 67 & 56,7 & 66 & 68,2 & 11,5 & 6 \\
\hline$S-5$ & 41 & 14,6 & 49 & 26,5 & 11,9 & 4 \\
\hline$S-48$ & 122 & 73,8 & 94 & 86,2 & 12,4 & 14 \\
\hline
\end{tabular}


Tab. 3 (Fortsetzung)

\begin{tabular}{|c|c|c|c|c|c|c|}
\hline $\begin{array}{l}\text { Schul- } \\
\text { bezirk/ } \\
\text { Schule }\end{array}$ & $\begin{array}{l}\text { Anzahl } \\
\text { gemel- } \\
\text { deter } \\
\text { Kinder }\end{array}$ & $\begin{array}{l}\text { Anteil der } \\
\text { Kinder mit } \\
\text { Migrations- } \\
\text { hintergrund } \\
\text { im Bezirk } \\
(\%)\end{array}$ & $\begin{array}{l}\text { Anzahl } \\
\text { einge- } \\
\text { schulter } \\
\text { Kinder }\end{array}$ & $\begin{array}{l}\text { Anteil der Kin- } \\
\text { der mit Migrati- } \\
\text { onshintergrund } \\
\text { in der Schule } \\
(\%)\end{array}$ & $\begin{array}{l}\text { Differenz } \\
\text { der Quo- } \\
\text { ten (in } \\
\text { Prozent- } \\
\text { punkten) }\end{array}$ & $\begin{array}{l}\text { Anzahl der } \\
\text { alternativ } \\
\text { angewähl- } \\
\text { ten Schulen }\end{array}$ \\
\hline$S-33-G L$ & 77 & 46,8 & 63 & 60,3 & 13,5 & 13 \\
\hline$S-6-G L$ & 71 & 45,1 & 58 & 58,6 & 13,5 & 9 \\
\hline$S-25$ & 71 & 28,2 & 67 & 43,3 & 15,1 & 7 \\
\hline$S-24$ & 102 & 62,7 & 60 & 86,7 & 24,0 & 18 \\
\hline$S-10-G L$ & 56 & 55,4 & 35 & 80,0 & 24,6 & 13 \\
\hline$S-17$ & 55 & 29,1 & 61 & 55,7 & 26,6 & 9 \\
\hline$F T-26$ & - & - & 49 & 36,7 & - & - \\
\hline$F T-35$ & - & - & 7 & 0,0 & - & - \\
\hline$F T-27-I$ & - & - & 56 & 0,0 & - & - \\
\hline$F T-20-I$ & - & - & 76 & 1,3 & - & - \\
\hline$F T-30-I$ & - & - & 5 & 0,0 & - & - \\
\hline$F-T-19$ & - & - & 29 & 3,4 & - & - \\
\hline$F T-21$ & - & - & 4 & 0,0 & - & - \\
\hline$F \ddot{O}-52$ & - & - & 1 & 0,0 & - & - \\
\hline$F \ddot{O}-51$ & - & - & 2 & 50,0 & - & - \\
\hline$F \ddot{O}-53$ & - & - & 1 & 100,0 & - & - \\
\hline$F \ddot{O}-54$ & - & - & 1 & 100,0 & - & - \\
\hline
\end{tabular}

Zur Anonymisierung wurden den Schulen und Bezirken randomisiert Zahlen zugeordnet. Bei Schulen mit einem Einzugsbezirk entspricht die Schulnummer der Schulbezirksnummer. Städtische Schulen sind mit dem Präfix S kodiert. Städtische Schulen mit Gemeinsamen Lernen sind zusätzlich mit dem Suffix GL kodiert. Nicht-städtische Schulen sind mit dem Präfix FT kodiert. Nicht-städtische Schulen mit ausgewiesen inklusiver Beschulung sind mit dem Suffix I kodiert. Förderschulen sind mit dem Präfix FÖ kodiert

Der Migrationshintergrund wird von den aufnehmenden Schulen erfasst. Was diese darunter verstehen, kann in der Praxis variieren. Er wird hier als eine Variable für die Fremdzuschreibung einer nicht ausschließlich deutschen Ethnizität verstanden

Für die Schulen in kirchlicher, privater oder sonstiger Trägerschaft und für die Förderschulen können nur die Größen der Einschulungsjahrgänge und der Anteil der Kinder mit einem gemeldeten Migrationshintergrund ausgewiesen werden, da diese Schulen an keinen Bezirk gebunden sind

\subsection{Homogenisierungseffekte bei breiter Abwanderung}

Im Folgenden wird ein Schulgeflecht vorgestellt, bei dem die eingangs beschriebenen Wanderungsbewegungen und Segregationseffekte in den Daten besonders auffällig werden. Bei Schule S-24 (in Tab. 3 markiert) kumulieren einige auffällige Effekte. Zum einen ist eine Abwanderungsbewegung zu 18 verschiedenen Schulen zu beobachten, also die mit Abstand höchste Verteilung. Zudem handelt es sich um den Bezirk mit dem höchsten Anteil an Kindern mit Migrationshintergrund (62,7\%). Der Anstieg des Anteils der Kinder mit Migrationshintergrund nach den Wanderungsbewegungen im Vergleich zur Startpartition um 24 Prozentpunkte ist der dritthöchste beobachtete Zuwachs (s. Tab. 3). Die Quote der Abwanderung aus dem Bezirk liegt bei $50 \%$. Das ist nicht der höchste Wert der Stichprobe - fünf weitere Bezirke 
Tab. 4 Übersicht über die am stärksten von Abwanderungsbewegungen betroffenen Schulen

\begin{tabular}{lll}
\hline Schule & $\begin{array}{l}\text { Anteil der Kinder, die den Einzugs- } \\
\text { bezirk verlassen } \\
\text { (in Prozent) }\end{array}$ & $\begin{array}{l}\text { Anteil der Kinder auf der Schule, die aus einem } \\
\text { anderen Schulbezirk stammen } \\
\text { (in Prozent) }\end{array}$ \\
\hline$S-24$ & $\mathbf{5 0 , 0}$ & 15,0 \\
$S-17$ & $\mathbf{5 0 , 9}$ & $\mathbf{5 5 , 7}$ \\
$S-10-G L$ & $\mathbf{5 1 , 8}$ & 22,9 \\
$S-8$ & $\mathbf{5 2 , 9}$ & 30,1 \\
$S-41$ & $\mathbf{5 4 , 7}$ & 21,6 \\
$S-46-G L$ & $\mathbf{6 5 , 8}$ & 28,9 \\
\hline
\end{tabular}

verzeichnen höhere Abwanderungsquoten (s. Tab. 4) - allerdings liegt die Zuwanderung von Schüler*innen zu Schule S-24 lediglich bei $15 \%$, der Bezirk wird also kaum ,aufgefüllt". Der starke beobachtete Homogenisierungseffekt in Bezug auf das Merkmal Migrationshintergrund und die gleichzeitige hohe Verteilung in weite Teile des Stadtgebiets machen diesen Bezirk bzw. diese Schule interessant für eine weitere Analyse.

Bei der Schule S-24 handelt es sich um einen Schulverbund mit 2 Standorten. Der Schulbezirk umfasst im untersuchten Jahrgang insgesamt 102 Schüler*innen. Bei näherer Analyse der Abwanderungsbewegung zeigen sich deutliche Auffälligkeiten Abb. 1 illustriert die Verteilung der wechselnden Kinder auf die bezirksgebundenen Schulen des Stadtgebiets. Tab. 5 schlüsselt die Anteile der Kinder mit einem zugeschriebenen Migrationshintergrund unter den wechselnden Kindern nach Zielschule auf. Elf der Zielschulen sind nicht in direkt angrenzenden Schulbezirken gelegen. Die größte zusammenhängende Gruppe von 10 Kindern wandert in den benachbarten Bezirk ab (S-47-GL), die zweitgrößte Gruppe von 6 Kindern wählt eine Schule in kirchlicher Trägerschaft (FT-26), die sich ebenfalls in räumlicher Nähe zu den Standorten, auf dem Gebiet des Einzugsbezirks S-37, befindet. In diesen beiden Gruppen ist bezüglich des Migrationshintergrundes eine vier zu sechs Verteilung in dem ersten Fall und eine Parität im zweiten Fall zu beobachten; damit sind Kinder mit Migrationshintergrund unter den Wechsler*innen an diese Schulen leicht überrepräsentiert. Eine Gruppe von 5 Kindern wandert zu einer Schule ab, die nahe an einer Ausfallstraße auf der anderen Seite der Innenstadt liegt (S-37). Obwohl es sich dabei um einen an den untersuchten Schulverbund angrenzenden Bezirk handelt, kann diese Wahl, aufgrund der stadtbaulichen Gegebenheiten (Trennung der Bezirke durch vielbefahrene Straßen), nicht gut durch räumliche Nähe und aufgrund der städtischen Trägerschaft auch nicht durch religiöse Bindung begründet werden. Auffällig ist, dass keinem der fünf Kinder, die Schule S-37 auswählen, ein Migrationshintergrund zugeschrieben wird (s. Tab. 5). Zwei weitere zusammenhängende Gruppen von jeweils vier Kindern wählen Schulen, die nicht an den eigenen Einzugsbezirk grenzen. Zum einen handelt es sich um eine Schule in sonstiger Trägerschaft, die im Einzugsgebiet S-49 liegt und zum anderen um eine städtische Schule (S-39). Unter den Wechsler*innen zur Schule in sonstiger Trägerschaft finden sich keine Kinder mit einem zugeschriebenen Migrationshintergrund, in der zweiten beschriebenen Gruppe ist die Verteilung paritätisch. Eine weitere Gruppe von 
Tab. 5 Übersicht über die Anzahl der Kinder, die in andere Schulen wechseln und den jeweiligen Anteil von Kindern mit einem gemeldeten Migrationshintergrund

\begin{tabular}{lll}
\hline $\begin{array}{l}\text { Angewählte } \\
\text { Schule }\end{array}$ & $\begin{array}{l}\text { Anzahl der } \\
\text { Kinder }\end{array}$ & $\begin{array}{l}\text { Anteil Kinder mit gemeldetem } \\
\text { Migrationshintergrund (\%) }\end{array}$ \\
\hline S49 & 1 & 100,0 \\
S48 & 1 & 0,0 \\
S28GL & 1 & 0,0 \\
S17 & 1 & 0,0 \\
S34GL & 1 & 0,0 \\
S42 & 1 & 0,0 \\
S11GL & 1 & 100,0 \\
S36GL & 2 & 50,0 \\
S33GL & 2 & 50,0 \\
S40GL & 3 & 33,3 \\
S10GL & 3 & 100,0 \\
S13 & 4 & 0,0 \\
S39 & 4 & 50,0 \\
FT27I & 4 & 0,0 \\
S37 & 5 & 0,0 \\
FT26 & 6 & 50,0 \\
S47GL & 10 & 60,0 \\
\hline
\end{tabular}

vier Kindern wechselt auf eine benachbarte städtische Schule, unter diesen Kindern befindet sich kein Kind mit zugeschriebenem Migrationshintergrund. Eine Ausnahme in der Häufung der Abwanderungen bildet eine Gruppe von drei Schüler*innen mit Migrationshintergrund, die gemeinsam eine andere innerstädtische Schule besuchet, in der ebenfalls ein hoher Migrationsanteil zu beobachten ist $(55,4 \%$ in der Ausgangspartition des Stadtteils, $80 \%$ bei Beginn des Schuljahres in der Schule; s. Tab. 3). Bei weiteren drei angewählten Schulen handelt es sich um Nachbarbezirke, hier liegt das Verhältnis in einem Fall bei eins zu zwei, in den beiden anderen bei einer paritätischen Verteilung. Sieben Kinder besuchen jeweils als einzige aus ihrem Bezirk eine andere Schule, davon haben zwei Kinder einen Migrationshintergrund. Ein Kind ohne Migrationshintergrund wechselt auf eine Schule außerhalb des Stadtbezirks. Insgesamt haben unter den wechselnden Kindern 37,3\% einen Migrationshintergrund. Es verlassen also weniger Kinder mit Migrationshintergrund als solche ohne Migrationshintergrund die Schule, obwohl sie in der Ausgangspartition überrepräsentiert sind.

Insgesamt zeigt sich eine Systematik bei der Betrachtung der abwandernden Kinder aufgeteilt nach der Zuschreibung eines Migrationshintergrunds in Bezug auf ihr Abwanderungs- bzw. Verbleibeverhalten, die in Abb. 2 illustriert ist. Während von den Kindern mit einem zugeschriebenen Migrationshintergrund lediglich 29,7\% eine andere Schule besuchen, liegt dieser Anteil bei Kindern ohne Migrationshintergrund bei $84,2 \%$. In absoluten Zahlen bedeutet das, dass lediglich sechs Kinder ohne Migrationshintergrund aus dem Schulbezirk auf der Schule verbleiben. Dieser Unterschied ist hoch signifikant $(p<0,001)$ und mit einem ausgewiesenen Phi- 


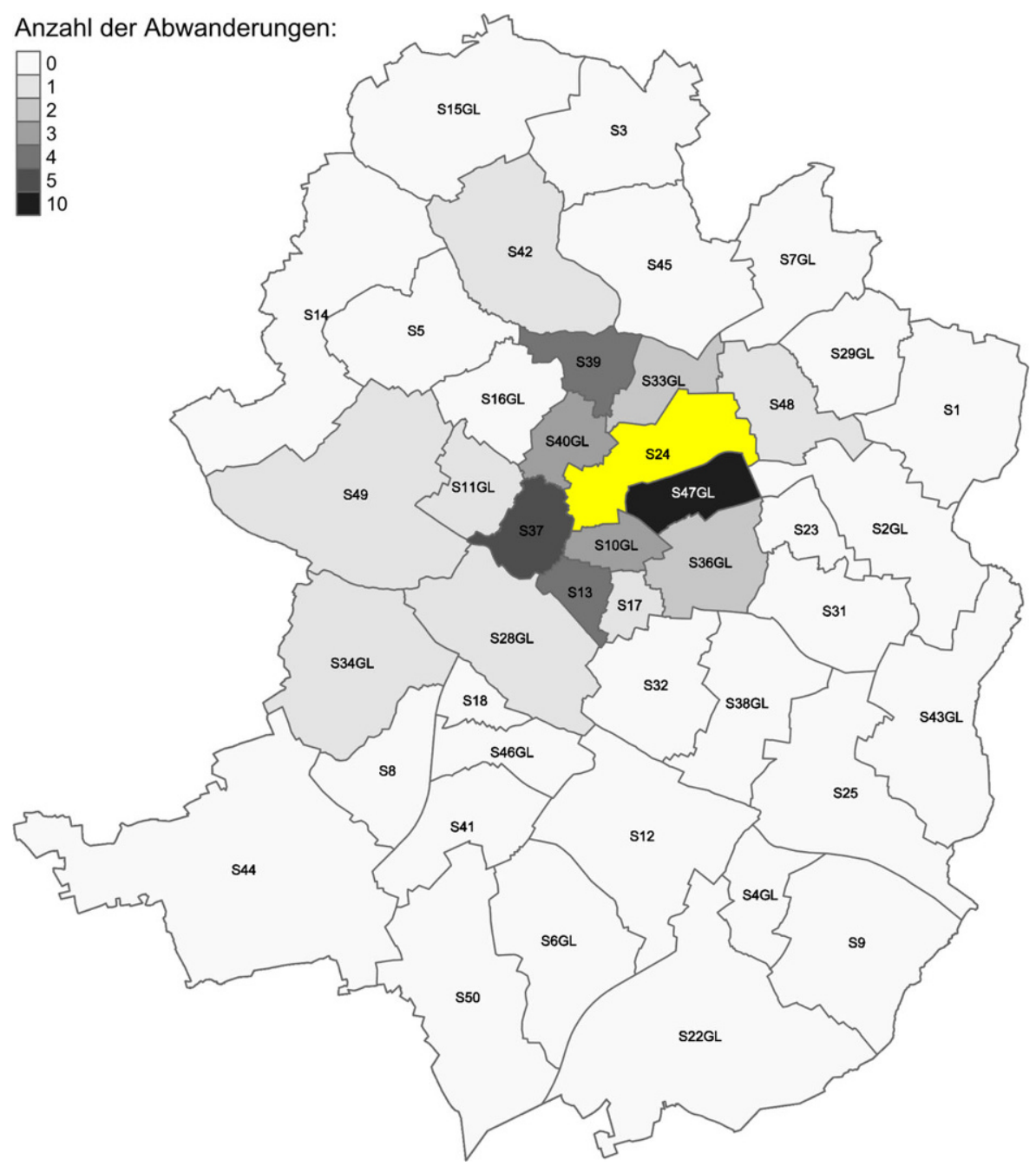

Abb. 1 Abwanderungsbewegungen aus Bezirk S-24 an bezirksgebundene Schulen. (Über die dargestellten bezirksgebundenen Schulen hinaus gab es aus dem Schulbezirk S-24 insgesamt zehn Abwanderungen zu Schulen in freier Trägerschaft und 1 Abwanderung durch Wegzug; die Karte wurde mithilfe des R-Pakets ,tmap“ erstellt)

Koeffizienten von 0,53 kann von einem starken Zusammenhang zwischen dem Migrationshintergrund und dem Abwanderungsverhalten ausgegangen werden.

Aus anderen Schulbezirken kommen neun Schüler*innen aus vier verschiedenen Bezirken des Stadtgebiets und einem außerstädtischen Bezirk (S-37, S-48, S-47GL, S-10-GL) zur Schule S-24, von denen zwei Drittel einen Migrationshintergrund zugeschrieben bekommen. Der Vergleich zwischen der Ausgangskomposition des Schulbezirks und der Komposition nach den Wechselbewegungen zeigt, dass sich die Quote der Kinder mit Migrationshintergrund stark verschoben hat und jetzt bei 86,7\% Kindern mit einem zugewiesenen Migrationsintergrund liegt. Somit zeigt sich 
Abb. 2 Anteile der wechselnden und verbleibenden Kinder aufgeschlüsselt nach dem zugeschriebenen Migrationshintergrund

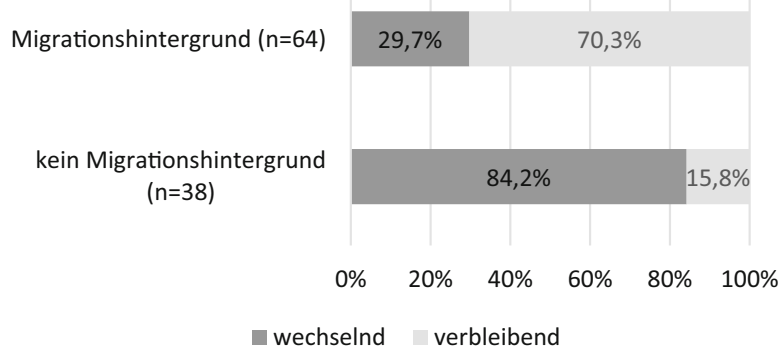

Migrationshintergrund $(n=64)$

als Folge der Wanderungsbewegungen eine Homogenisierung der Schülerschaft in Bezug auf das Merkmal Migrationshintergrund in dem fokussierten Bezirk.

\section{Diskussion}

Insgesamt konnten in der untersuchten Stadt starke Wanderungsbewegungen aufgezeigt und ein Schulgeflecht illustriert werden. Die durchgeführte explorative Untersuchung stand unter der Frage, ob und in welche Richtung die unter den Bedingungen freier Schulwahl möglichen Wanderungsbewegungen zwischen den Schulbezirken die Kompositionen der Einzelschulen verändern. Das dargestellte Beispiel weist auf eine Systematik hinter den $\mathrm{Zu}$ - und Abwanderungsprozessen hin, die die Komposition des Einschulungsjahrgangs im Hinblick auf die ethnisch-kulturelle Dimension verändert und zu einer Aggregation des von der aufnehmenden Schule zugeschriebenen Merkmals Migrationshintergrund führt. Eine Limitation hinsichtlich der Datenbasis ist, dass die Angaben zum Migrationshintergrund aus dem Datenbestand der aufnehmenden Schulen stammen; die konkreten Zuschreibungspraxen sind jedoch bisher nicht im Detail bekannt. Möglicherweise ist nicht einheitlich, wie die Schulen - trotz der in Abschn. 3.3 beschriebenen Basierung auf den Elternangaben zur Erstsprache - einen Migrationshintergrund festlegen. Wir gehen jedoch davon aus, dass diese Zuschreibung hinreichend valide ist, um zu dem Schluss zu kommen, dass sich die Schülerschaft durch die beschriebenen Wanderungsbewegungen innerhalb des Schulgeflechtes nach ethnisch-kulturellen Merkmalen segregiert.

Diese Segregation hat Folgen: Aus Befunden empirischer Bildungsforschung geht hervor, dass durch Kompositionseffekte, bei denen überdurchschnittlich viele Kinder aus deprivilegierten Familien in Einschulungsjahrgängen zusammenkommen, Nachteile entstehen können. Diese Nachteile werden, den meisten Studien folgend, größtenteils durch soziale Risikofaktoren und Unterschiede im Fähigkeits- und Leistungsniveau erklärt (Dumont et al. 2013), jedoch finden sich auch Hinweise auf einen spezifischen Effekt der ethnischen Zusammensetzung von Klassen bzw. Schulen (Van Ewijk und Sleegers 2010). Darüber hinaus wird Inklusion im Sinne von Normalisierung (Boger 2015, S. 52f.) eingeschränkt, zumal die Problemlagen in diesen Schulen häufig kumulieren (z. B. Baumert et al. 2003). Diesem Befund steht andererseits die Überlegung entgegen, dass eine Schule, die eine homogene Klientel 
rekrutiert, auf die spezifischen Bedarfe dieser Klientel besser eingehen kann und so möglicherweise Prozesse von Empowerment stattfinden. Es stellen sich also die grundlegenden Fragen, ob diese Segregationseffekte a) auf eine Einschränkung von Verwirklichungschancen durch systematische Exklusion vom freien Zugang zu frei gewählten Bildungseinrichtungen hindeuten (Nussbaum 2007), ob b) ein habituell bedingtes Streben nach Homologie und somit die spezifische Logik des Feldes wirkmächtig wird (Bourdieu 1982) oder c) im Gegenteil zielgenaue Unterstützungsmaßnahmen zur Verbesserung der Befähigungssituation der Kinder zu dem beobachteten Wahlverhalten führen, was dann vor dem Hintergrund des Trilemmas der Inklusion (Boger 2015, S. 54) als Empowerment zu interpretieren wäre. Diese Fragen stellen die Grundlage der Weiterarbeit der Forschungsgruppe dar.

Funding Open Access funding enabled and organized by Projekt DEAL.

Open Access Dieser Artikel wird unter der Creative Commons Namensnennung 4.0 International Lizenz veröffentlicht, welche die Nutzung, Vervielfältigung, Bearbeitung, Verbreitung und Wiedergabe in jeglichem Medium und Format erlaubt, sofern Sie den/die ursprünglichen Autor(en) und die Quelle ordnungsgemäß nennen, einen Link zur Creative Commons Lizenz beifügen und angeben, ob Änderungen vorgenommen wurden.

Die in diesem Artikel enthaltenen Bilder und sonstiges Drittmaterial unterliegen ebenfalls der genannten Creative Commons Lizenz, sofern sich aus der Abbildungslegende nichts anderes ergibt. Sofern das betreffende Material nicht unter der genannten Creative Commons Lizenz steht und die betreffende Handlung nicht nach gesetzlichen Vorschriften erlaubt ist, ist für die oben aufgeführten Weiterverwendungen des Materials die Einwilligung des jeweiligen Rechteinhabers einzuholen.

Weitere Details zur Lizenz entnehmen Sie bitte der Lizenzinformation auf http://creativecommons.org/ licenses/by/4.0/deed.de.

\section{Literatur}

van Ackeren, I., \& Klemm, K. (2019). 100 Jahre Grundschule - Soziale Chancenungleichheit und kein Ende. Zeitschrift für Grundschulforschung, 12(2), 399-414. https://doi.org/10.1007/s42278-01900057-4.

Aytekin, V., \& Boger, M.-A. (2016). Subalterne Vorstellungen von Bildung. In S. Geuenich, D. KrenzDewe \& D. Niggemann (Hrsg.), Wozu brauchen wir das? - Bildungsphilosophie und pädagogische Praxis (S. 112-122). Münster: Westfälisches Dampfboot.

Baumert Jürgen, Trautwein, U., \& Cordula, A. (2003). Schulumwelten - institutionelle Bedingungen des Lehrens und Lernens. In J. Baumert, et al. (Hrsg.), PISA 2000 - Ein differenzierter Blick auf die Länder der Bundesrepublik Deutschland. Wiesbaden: VS. https://doi.org/10.1007/978-3-322-975904 _11.

Bildungsberichterstattung, Autorengruppe. (2016). Bildung in Deutschland 2016: Ein indikatorengestützter Bericht mit einer Analyse zu Bildung und Migration. : Bertelsmann.

Boger, M.-A. (2015). Theorie der trilemmatischen Inkluison. In I. Schnell (Hrsg.), Herausforderung Inklusion. Theoriebildung und Praxis (S. 51-62). Bad Heilbrunn: Klinkhardt.

Boger, M.-A., \& Störtländer, J.C. (2019). Lehrerhandeln aus der Sicht von Schüler*innen in existentiellen Krisen Vier Fallstudien zwischen falschem Mitleid und falscher Härte. In S. Drucks \& D. Bruland (Hrsg.), Kritische Lebensereignisse und die Herausforderungen für die Schule (S. 91-105). Weinheim: Beltz Juventa.

Boger, M.-A., \& Textor, A. (2016). Das Förderungs-Stigmatisierungs-Dilemma, Oder: Der Effekt diagnostischer Kategorien auf die Wahrnehmung durch Lehrkräfte. In B. Amrhein (Hrsg.), Diagnostik im Kontext inklusiver Bildung. Theorien, Ambivalenzen, Akteure, Konzepte (S. 79-97). Bad Heilbrunn: Klinkhardt.

Bourdieu, P. (1982). Die feinen Unterschiede. Frankfurt am Main: Suhrkamp. 
Clausen, M. (2006). Warum wählen Sie genau diese Schule? Eine inhaltsanalytische Untersuchung elterlicher Begründungen der Wahl der Einzelschule innerhalb eines Bildungsgangs. Weinheim: Beltz.

Dumont, H., Neumann, M., Maaz, K., \& Trautwein, U. (2013). Die Zusammensetzung der Schülerschaft als Einflussfaktor für Schulleistungen. Psychologie in Erziehung und Unterricht, 60(3), 163-183. https://doi.org/10.2378/peu2013.art14.

van Ewijk, R., \& Sleegers, P. (2010). Peer ethnicity and achievement: a meta-analysis into the compositional effect. School Effectiveness and School Improvement, 21(3), 237-265. https://doi.org/10.1080/ 09243451003612671.

Fincke, G., \& Lange, S. (2012). Segregation an Grundschulen: Der Einfluss der elterlichen Schulwahl. Berlin: Sachverständigenrat deutscher Stiftungen für Integration und Migration GmbH (SVR).

Gold, J. (2018). Obligatorische und fakultative Fördersysteme an der Versuchsschule Oberstufen-Kolleg Bielefeld und ihre Nutzung durch die Kollegiatinnen und Kollegiaten. Eine empirische Untersuchung aus der Capability Perspektive. Bielefeld:

Hogrebe, N. (2016). Segregation am Übergang von der Kita zur Grundschule. In R. Strietholt, et al. (Hrsg.), Daten, Beispiele und Perspektiven. Jahrbuch der Schulentwicklung, (Bd. 19, S. 84-109). Weinheim, Basel: Beltz Juventa.

Köller, O., et al. (2019). Das Bildungswesen in Deutschland. Stuttgart: UTB; Klinkhardt.

Kölm, J., Greesch, C., \& Haag, N. (2017). Hintergrundmerkmale von Schülerinnen und Schülern mit sonderpädagogischem Förderbedarf an Förderschulen und an allgemeinen Schulen. In P. Stanat (Hrsg.), IQB-Bildungstrend 2016. Kompetenzen in den Fächern Deutsch und Mathematik am Ende der 4. Jahrgangsstufe im zweiten Ländervergleich (S. 291-301). Münster, New York: Waxmann.

Krüger, J. O., Roch, A., \& Breidenstein, G. (2020). Szenarien der Grundschulwahl: Eine Untersuchung Von Entscheidungsdiskursen Am Übergang Zum Primarbereich. Wiesbaden: Springer VS.

Lindmeier, C., \& Lütje-Klose, B. (2015). Inklusion als Querschnittsaufgabe in der Erziehungswissenschaft. Erziehungswissenschaft, 26(51), 7-16.

Maaz, K. (2017). Chancengerechtigkeit. Ein Ding der (Un-)Möglichkeit? News und science, 44(2), 41-44.

Maaz, K., Baumert, J., \& Trautwein, U. (2010). Genese sozialer Ungleichheit im institutionellen Kontext der Schule: Wo entsteht und vergrößert sich soziale Ungleichheit? In J. Baumert, K. Maaz \& U. Trautwein (Hrsg.), Bildungsentscheidungen (S. 11-46). Wiesbaden: VS.

Makles, A., \& Schneider, K. (2013). Much Ado about nothing? The role of primary school catchment areas for ethnic school segregation. Evidence from a policy reform. CESifo working paper no. 4520, category 5: economics of education. https:/www.ifo.de/DocDL/cesifo1_wp4520.pdf. Zugegriffen: 18. Mai 2021.

Makles, A., Schneider, K., \& Terlinden, B. (2019). Schulische Segregation und Schulwahl. Eine Analyse mit den Daten der Bremer Schülerindividualstatistik. In D. Fickermann \& H. Weishaupt (Hrsg.), Bildungsforschung mit Daten der amtlichen Statistik (S. 176-196). Münster, New York: Waxmann.

Nussbaum Craven, M. (2007). Frontiers of justice. Disability, nationality, species membership. The Tanner lectures on human values. Cambridge, Massachusetts, London: Belknap Press.

Nussbaum Craven, M. (2011). Creating capabilities. The human development approach. Harvard: Harvard University Press.

Nussbaum Craven, M. (2016). Gerechtigkeit oder Das gute Leben (9. Aufl.). Gender studies, Bd. 739. Frankfurt am Main: Suhrkamp.

Rjosk, C., Haag, N., Heppt, B., \& Petra, S. (2017). Zuwanderungsbezogene Disparitäten. In P. Stanat, S. Schipolowski, C. Rjosk, S. Weirich \& N. Haag (Hrsg.), IQB-Bildungstrend 2016. Kompetenzen in den Fächern Deutsch und Mathematik am Ende der 4. Jahrgangsstufe im zweiten Ländervergleich (S. 237-275). Münster, New York: Waxmann.

Sen, A. K. (2011). The idea of justice. Cambridge: Belknap Press of Harvard Univ. Press.

Stanat, P., Schwippert, K., \& Gröhlich, C. (2010). Der Einfluss des Migrantenanteils in Schulklassen auf den Kompetenzerwerbs. Längsschnittliche Überprüfung eines umstrittenen Effekts. In C. AllemannGhionda, P. Stanat, K. Göbel \& C. Röhner (Hrsg.), Migration, Identität, Sprache und Bildungserfolg (S. 147-164). Weinheim: Beltz Juventa.

Störtländer, J.C. (2019). Bildung und Befähigung. Eine qualitative Studie zu kritisch-konstruktiver Didaktik und Capabilities Approach. Weinheim: Beltz Juventa.

Textor, A. (2018). Einführung in die Inklusionspädagogik. Bad Heilbrunn: Julius Klinkhardt.

Wolff, J., \& de-Shalit, A. (2007). Disadvantage. Oxford: Oxford University Press. 